\title{
Longitudinal Comparison of Two Severities of Unilateral Cervical Spinal Cord Injury Using Magnetic Resonance Imaging in Rats
}

\author{
GEORGETA MIHAI, ${ }^{1}$ YVETTE S. NOUT, ${ }^{2,3}$ C. AMY TOVAR, \\ BRANDON A. MILLER, ${ }^{2,3}$ PETRA SCHMALBROCK, \\ JACQUELINE C. BRESNAHAN, ${ }^{2,3}$ and MICHAEL S. BEATTIE ${ }^{2,3}$
}

\begin{abstract}
Magnetic resonance imaging (MRI) should be a powerful tool for characterization of spinal cord pathology in animal models. We evaluated the utility of medium-field MRI for the longitudinal assessment of progression of spinal cord injury (SCI) in a rat model. Thirteen adult rats were subjected to a 6.25 or $25 \mathrm{~g}-\mathrm{cm}$ unilateral cervical SCI, and underwent MRI and behavioral tests during a 3-week study period. MRI was also performed post-mortem. Quantification of cord swelling, hypointense and hyperintense signal, and lesion length were the most valuable parameters to determine and were highly correlated to behavioral and histopathological measures. Immediately after injury, MRI showed loss of gray matter-white matter differentiation, presence of scattered hyperintense signal and local hypointense signal, and cord swelling in both groups. At 7 days after injury, the spinal cord in the $25 \mathrm{~g}-\mathrm{cm}$ group was significantly larger than that of the $6.25 \mathrm{~g}-\mathrm{cm}$ group $(p=$ 0.02). Contrast enhancement of the lesion was seen at $24 \mathrm{~h}$ in the $6.25 \mathrm{~g}-\mathrm{cm}$ group, and at $24 \mathrm{~h}$ and 7 days in the $25 \mathrm{~g}-\mathrm{cm}$ group. The volume of hypointense signal, representing hemorrhage, throughout the lesion region was significantly larger in the $25 \mathrm{~g}-\mathrm{cm}$ compared to the $6.25 \mathrm{~g}-\mathrm{cm}$ group at both 14 and 21 days after SCI $(p<0.04)$. The appearance of the scattered hyperintense signal, initially representing edema, at later time points changed to a rim of hyperintense signal surrounding the lesion cavity. Significant correlations were found between cord swelling at 7 days after SCI, and lesion length and gray and white matter sparing as determined by histopathology. Other parameters that were highly correlated with histopathology were quantity of hyperintense and hypointense signal, and in vivo lesion length. Hypointense signal and in vivo lesion length were highly correlated to behavior. Significant correlation was also found between parameters determined by MRI: swelling, hypointense signal, hyperintense signal, and lesion length. MRI is a valuable imaging modality to assess the temporal evolution of SCI and to distinguish different severities of cervical SCI in rats. In future, MRI could be applied as a screening tool to either administer goal-directed therapies, or enable even group distribution, prior to therapeutic intervention for example through quantification and matching of swelling and edema.
\end{abstract}

Key words: secondary injury

Departments of ${ }^{1}$ Radiology and ${ }^{2}$ Neuroscience, The Ohio State University, Columbus, Ohio.

${ }^{3}$ Brain and Spinal Injury Center, Department of Neurological Surgery, University of California, San Francisco, San Francisco, California. 


\section{MIHAI ET AL.}

\section{INTRODUCTION}

$\mathbf{S}$ PINAL CORD INJURY (SCI) is a debilitating and costly condition. In human SCI, injuries to the cervical region of the spinal cord are the most common (42.9\%), and estimated lifetime costs reach USD 1.2-2.2 million (National Spinal Cord Injury Statistical Center, 2006). In order to study cervical SCI specifically, a number of rodent animal models have recently been developed (Gensel et al., 2006; Pearse et al., 2005; Soblosky et al., 2001); however, in vivo imaging studies of this region of the spinal cord in the rat are limited (Ohta et al., 1999).

Magnetic resonance imaging (MRI) has shown promise as a useful and powerful tool for detection and characterization of spinal cord pathology in animal models (Berens et al., 2005; Bilgen et al., 2000, 2001b; Bilgen and Narayana, 2001; Chakeres et al., 1987; Deo et al., 2006; Falconer et al., 1994; Narayana et al., 1999, 2004; Ohta et al., 1999; Schwartz and Hackney, 2003; Weber et al., 2006). The noninvasive nature of MRI offers the possibility of following pathological changes over time in the same animal, avoids inter-subject variability, and decreases the number of animals that need to be used to attain statistical significance. Previously published reports are mostly descriptive, and compare histopathological and MRI findings of spinal cord pathology, and, although some quantitative analyses are available, we hypothesize that focused quantification of MRI data and analysis of changes over time will greatly increase our understanding of the events that follow SCI. In particular, we expect to use MRI to characterize events that occur during the phase of secondary injury, such as development of hemorrhage and edema, and hypothesize that changes detectable by MRI may predict long term histopathological and behavioral outcomes. Indeed, MRI findings in human cervical SCI correlate with neurological outcome (Boldin et al., 2006; Miyanji et al., 2007; Selden et al., 1999).

Exploring the use of MRI in a cervical hemicontusion model, such as the one described by Gensel et al. (2006), has so far not been reported, and carries some distinct advantages over MRI in thoracic SCI. First, the size of the cervical spinal cord is larger than that of the thoracic spinal cord (width by height: $4.3 \times 3.2 \mathrm{~mm}$ and $3.0 \times$ $2.4 \mathrm{~mm}$, respectively) (Waibl, 1973). Second, cervical spine MRI is not as heavily affected by motion artifacts induced by normal respiration as is the thoracic column. The small dimensions of the rat spinal cord (small field of view) require MRI techniques that allow for fairly high spatial resolution without an excessively long scan time. In order to differentiate and characterize very small structures inside the rodent spinal cord, higher magnetic fields, implantable coils, or other experimental MRI techniques, have been used (Bilgen et al., 2001b; Elshafiey et al., 2002; Gareau et al., 2001; Silver et al., 2001). Investigating MRI in a cervical SCI rat model is less technically challenging, requiring less invasive techniques when compared to using a thoracic SCI model.

The aims of this study are to determine whether MRI can differentiate between two injury severities in the rat, and to further characterize the pathological evolution of cervical SCI over time. MRI, behavioral analysis, and histopathology are used to monitor rats during a 3-week study period after mild and severe unilateral cervical contusion SCI. Thus, two different levels of cervical SCI are compared longitudinally, and quantitative in vivo MRI data are correlated to neurobehavioral testing, post mortem MRI, and histology. The results suggest that MRI can be a useful addition to the evaluation of this cervical injury model by adding longitudinal sequential measures of secondary injury dynamics. This is intended to provide a basis for the evaluation of acute and subacute therapies that can then be evaluated in similar fashion in human SCI.

\section{METHODS}

\section{Subjects}

Thirteen adult, female Long-Evans hooded rats ( $\mathrm{Si}-$ monsen Laboratories, Gilroy, CA), age 84 days (range, 83-86 days), were used in this study. Rats were housed individually in plastic cages, maintained on a 12-h light/dark cycle, and had access to food and water ad libitum. All animal experiments were conducted after approval by the Institutional Laboratory Animal Care and Use Committee of The Ohio State University, and were performed in compliance with NIH guidelines and recommendations.

\section{Surgical Procedures and Post-Operative Care}

Surgical procedures were carried out aseptically under deep anesthesia induced by intraperitoneal (IP) administration of pentobarbital (Abbott Laboratories, Chicago, IL; $55 \mathrm{mg} / \mathrm{kg}$ ). Anesthetic plane was determined by foot pinch. A pre-operative dose of cefazolin (Ancef, Abbott Laboratories, N. Chicago, IL; $50 \mathrm{mg} / \mathrm{kg}$ ) was administered subcutaneously (sc). Lacrilube ophthalmic ointment (Allergan Pharmaceuticals, Irvine, CA) was applied to the eyes prior to surgery and body temperature was monitored using a rectal thermal probe and maintained at $37.5 \pm 0.5^{\circ} \mathrm{C}$ using a heating pad.

A catheter (PE-50-polyethylene tubing, Clay Adams, Division of Becton-Dickinson Co., Parsippany, NJ) was placed in the right jugular vein. The intravenous (IV) tub- 


\section{MRI IN LONGITUDINAL COMPARISON OF UNILATERAL CERVICAL SPINAL CORD INJURIES}

ing was tunneled subcutaneously to the back where it protruded from the rostral aspect of the dorsal midline incision for easy access. The free end of the PE-50 tubing was heat-sealed and kept in place with a skin staple.

A 6.25 - or 25-mm drop height, unilateral injury at the level of cervical vertebra 5 (C5) was produced as previously described (Gensel et al., 2006). Briefly, a dorsal midline incision was made and a dorsal laminectomy at C5 was performed to expose the entire right side and most of the left side of the spinal cord. A right-sided contusion injury was produced using a MASCIS/NYU injury device (Gruner, 1992) with a modified 2.0-mm-diameter impounder rod. The rod was centered over the right side of the spinal cord so that the medial curve was aligned with the midline tangent. The spinal cord, with the dura mater intact, was impacted with the $10 \mathrm{-g}$ rod from a height of $6.25 \mathrm{~mm}(n=6)$ or $25 \mathrm{~mm}(n=7)$. After injury, the muscle layers were closed, the skin was apposed with skin staples, and animals were allowed to recover in an incubator $\left(37^{\circ} \mathrm{C}\right)$ for $24 \mathrm{~h}$ post-operatively, after which they were returned to their home cages. Post-operative care included continued administration of cefazolin sc for 5 days, and once daily animal check throughout the remainder of the study. Furthermore, animals were administered lactated Ringer's solution sc (Abbott Laboratories, North Chicago, IL) if there was evidence of dehydration, and nutritional supplementation (NutriCal: Evsco Pharmaceuticals, Division of VÇtoquinol USA Inc., Buena, NJ) if animals lost more than $10 \%$ of bodyweight. Bladder care was not required for this injury.

\section{Behavioral Analysis}

Open-field locomotor testing. Open-field walking was evaluated before SCI and at 2, 4-6, 8, 15, and 22 days following SCI using the 21-point Basso Beattie Bresnahan (BBB) Locomotor Rating Scale (Basso et al., 1995) and the 13-point BBB subscoring system (Popovich et al., 1999).

Grooming test. Forelimb grooming function was assessed as previously described (Bertelli and Mira, 1993; Gensel et al., 2006). Animals were placed in a clear plastic cylinder with two mirrors placed at angles such that the rat's head was always clearly visible. Cool tap water was applied to the animal's head and back with soft gauze prior to placing the animal in the cylinder, and the animal was recorded with a digital camera for $5 \mathrm{~min}$. Slow motion DVD playback was used to score each forelimb independently by the maximal contact made while performing any part of the grooming sequence. A six-point scoring system was used in which 0 indicates an animal's inability to contact any part of the face or head; 1 indicates the animal's forepaw making contact with the underside of the chin and/or mouth area; 2 indicates the animal's forepaw contacting the area between nose and eyes, but not the eyes; 3 indicates the animal's forepaw contacting the eyes and the area up to, but not including, the front of the ears; 4 indicates the animal's forepaw contacting the ears, but not the area of the head behind the ears; and 5 indicates the animal's forepaw contacting the area of the head behind the ears. Animals were tested before SCI, and at 48 h, 8 days, 15 days, and 22 days after SCI.

Paw preference test. The forelimb asymmetry, or paw preference test, was performed as previously described (Gensel et al., 2006; Schallert et al., 1986). Animals were placed in a clear plastic cylinder with two mirrors placed at angles such that all sides of the rat were clearly visible. The animal was recorded with a digital camera for 5 min, and slow motion DVD playback was used to determine the number of the times the animal placed its left, right, or both forepaws against the side of the cylinder during weight supported movements. Individual placements were scored as either "left" or "right" when 0.5 sec or more passed without the other limb contacting the side of the cylinder. If both forepaws were used for weight supported movements within $0.5 \mathrm{sec}$ of each other, a score of "both" was given. During lateral exploration, a "both" score was given for every two-step "walking" sequence, during which, both paws changed position on the side of the cylinder. If one paw remained anchored while the other was placed on different parts of the cylinder, no score was given until the anchored forepaw was lifted. Animals were tested before SCI and on day 23 after SCI.

\section{MRI Protocol}

For MRI, rats were anesthetized with inhalant Isoflurane (maintenance: 1-2\%). Lacrilube ophthalmic ointment was applied to the eyes and all skin staples were removed. For administration of gadodiamide IV (Ominscan, Amersham Health Inc., Princeton, NJ; 0.1 $\mathrm{mmol} / \mathrm{kg}$ ), the heat sealed PE-50 tubing was cut and connected to a longer PE-50 tubing that contained $0.2 \mathrm{~mL}$ of diluted (1:4) contrast agent and $0.3 \mathrm{~mL}$ of $\mathrm{NaCl}(0.9 \%)$ to flush. This allowed for administration of contrast agent from outside the MRI system without the need to move the animal.

Each rat was imaged prior to cervical SCI, and at 24 h, and 7, 14, and 21 days post-injury, using a Bruker 4.7Tesla/40cm horizontal bore MRI System, with a 400$\mathrm{mT} / \mathrm{m}, 120-\mathrm{mm}-\mathrm{ID}$ (inner diameter) gradient insert. A 


\section{MIHAI ET AL.}

laboratory-built 3-cm-diameter Helmholtz coil was used for transmitting and receiving the MRI signal. Rats were placed in prone position (ventral recumbency) on a laboratory-built Plexiglas holder that also incorporated the matching and tuning electronic circuitry of the radiofrequency (RF) coil. The cervical region of the animal was placed between and parallel to the two loops of the RF coil, and fixed in place with masking tape. Exterior landmarks (occipital bone and shoulder blades) were used to ensure C5 was placed at the center of the coil. Injury location (C5) was carefully checked for positioning at the isocenter of both the RF antenna and within the magnet. Tuning and matching of the Helmholtz coil was performed every time for each of the animals, prior to and after insertion into the magnet. Acquisition of a three plane localizer for identifying the vertebral column was followed by a higher resolution 2.2-min sagittal gradient echo, T1-weighted localizer $(\mathrm{TR} / \mathrm{TE}=500 / 4.5 \mathrm{msec}$; flip angle $=90$ degrees) that allowed depiction of vertebral bodies, which were used as anatomical landmarks to achieve reproducible placement of axial scans for followup studies. Axial MRI studies covered a 16.4-mm region of the spinal cord, starting just rostral to the second thoracic spinous process, and extending from the third cervical to the first thoracic vertebra.

The high resolution MRI protocol included axial and sagittal T1-weighted (gradient echo: TR/TE/Flip angle $=$ $500 / 4.9 \mathrm{msec} / 90^{\circ}, 5$ averages; scan time $=10 \mathrm{~min}, 40$ $\mathrm{sec}$ ) and proton density-weighted (PD) (spin echo: $\mathrm{TR} / \mathrm{TE}=2000 / 15 \mathrm{msec}, 2$ averages; $\mathrm{scan}$ time $=17 \mathrm{~min}$, $7 \mathrm{sec}$ ) images acquired with $175 \times 175 \mu \mathrm{m}$ in-plane resolution and 1-mm slice thickness with 0.1-mm gap between slices. In order to assess blood-spinal cord barrier (BSCB) integrity, T1-weighted images were acquired before and after IV administration of gadodiamide. In addition, three-dimensional (3D) sagittal T2 (fast spin echo, TR/TE/RARE factor $=1630 \mathrm{msec} / 60 \mathrm{msec} / 16,2$ averages; scan time $=27 \mathrm{~min}, 48 \mathrm{sec}$ ) were acquired with $182 \times 175 \times 625 \mu \mathrm{m}$ resolution. Also, for one $25 \mathrm{~g}-\mathrm{cm}$ injured rat and all $6.25 \mathrm{~g}-\mathrm{cm}$ injured animals, axial 3D T2 images were acquired with the same acquisition parameters as the sagittal 3D T2 weighted images.

After MRI, the IV catheter was heat-sealed at the rostral end of the dorsal midline incision and secured with a skin staple. The skin was re-apposed with staples as needed and the animal was allowed to recover from anesthesia.

\section{Post-Mortem Analysis}

Sacrifice. At 23 days after SCI, animals were anesthetized with xylazine (TranquiVed ${ }^{\mathrm{TM}}$, Vedco Inc., St. Joseph, $\mathrm{MO} ; 10 \mathrm{mg} / \mathrm{kg} \mathrm{IP}$ ) and ketamine (ketamine $\mathrm{HCl}$, Abbott Laboratories, N. Chicago, IL; $80 \mathrm{mg} / \mathrm{kg}$ IP), and they were transcardially perfused with $0.9 \% \mathrm{NaCl}$ and $4 \%$ paraformaldehyde in phosphate-buffered saline (PBS).

MRI ex vivo in situ. The intact fixed animal was imaged using a $10-\mathrm{cm}$-diameter volume transmitter coil and a 5-cm-diameter surface receiver coil. A 3D T2 axial sequence (fast spin echo, TR/TE/RARE factor $=1630 / 63$ msec/16, 15 averages; scan time $=14 \mathrm{~h}$ ) with a resolution of $195 \times 195 \times 195 \mu \mathrm{m}$ was used to obtain isotropic high spatial resolution images of the spinal cord.

MRI ex vivo. The injured region of the cervical spinal cord was removed from the animal and post-fixed in $4 \%$ paraformaldehyde for $>48 \mathrm{~h}$. Cords were embedded in $2 \%$ agar with $1 \%$ sodium azide. In a petri-dish, a layer of $7.5 \mathrm{~mL}$ of agar was poured, and cords were secured on top of the hardened agar and then covered with an additional $7.5 \mathrm{~mL}$ of agar. MRI of the embedded cords was performed using the same technique as described above for the ex vivo in situ MRI.

Histopathological analysis. Cords were removed from the agar and cryoprotected in $30 \%$ sucrose in PBS for $48-72 \mathrm{~h}$. The tissue was frozen at $-80^{\circ} \mathrm{C}$ until further analysis. Lesion regions $(10 \mathrm{~mm})$ were sectioned transversely at $20 \mu \mathrm{m}$ on a cryostat. Sections were stained with Luxol fast blue for myelin and counterstained with cresyl echt violet for Nissl substance. Lesion length, area of lesion cavity at lesion epicenter, and sparing of white and gray matter as a percentage of the contralateral white and gray at the lesion epicenter were determined for each animal from these sections. In addition, large motor neurons (diameter: 25-70 $\mu \mathrm{m}$ ) with a discernible nucleus that were present in the ventral gray matter were counted in one section at the lesion epicenter for all animals.

\section{Quantitative MRI Analysis}

Axial MR images were used to quantify the evolution of the pathology in the two SCI groups. Axial images were reconstructed from the raw data, magnified four times, and cropped to retain just the vertebral column using IDL (Research System Inc., Boulder, CO) and saved for further processing using Adobe Photoshop 5.5 software (Adobe System Inc., San Jose, CA).

Ten matching axial images covering a length of $11 \mathrm{~mm}$ centered at the lesion epicenter from the PD, and pre- and post-contrast T1 scans were superimposed using Adobe Photoshop 5.5 software (Adobe System Inc.). Regions of interest were manually traced by a blinded reader, experienced with spinal cord anatomy. Areas of the right and left hemicord, whole cord, and hypointensity within the ipsilateral cord were determined. Photoshop pixel counts 
were converted into area units $\left(\mathrm{mm}^{2}\right)$ by scaling with the in-plane pixel size. Volume measurements $\left(\mathrm{mm}^{3}\right)$ were obtained by adding the individual slice areas and multiplying by $1.1 \mathrm{~mm}$ slice plus gap thickness.

Images from axial 3D T2-weighted MR images of one $25 \mathrm{~g}-\mathrm{cm}$ injured rat and all $6.25 \mathrm{~g}-\mathrm{cm}$ injured rats were used to assess and compare the evolution of hyperintense signal using MetaMorph vs.6.3 software (Molecular Devices Corporation, Downingtown, PA). The area of hyperintense signal was determined for 14 consecutive slices centered at the injury epicenter by implementing a threshold for the pixel intensities, based on the visual spread of hyperintensity. Areas were multiplied by the slice thickness, $0.625 \mathrm{~mm}$, to obtain the spread of hyperintensity over a length of $8.25 \mathrm{~mm}$ (i.e., 14 slices).

The presence of gadodiamide within the injured spinal cord was initially determined by visual inspection of the images. Subsequently, penetration of gadodiamide into the spinal cord was determined by subtracting each axial T1-weighted image obtained prior to contrast administration, from the one obtained after contrast admin- istration, using IDL. This allowed for improved visualization of enhanced regions.

\section{Statistical Analysis}

Quantitative MRI data, histopathology measurements, and behavioral data are presented as means \pm standard error (SE) for all rats in each injury level group. A factorial repeated measures analysis of variance (ANOVA) was used to analyze all behavioral data. The null hypothesis was rejected at $\alpha=0.05$. A $t$-test was used to compare endpoint data (day 21 and post-mortem) between the two groups of animals. Significant differences identified by the ANOVA were isolated using the HolmSidak procedure for pairwise multiple comparison posthoc test. To examine the relationship between quantitative MRI data, behavior, and histology, the Pearson Produce Moment Correlation Coefficient was determined. Statistical computations were performed with software packages Sigmastat 3.0 (SPSS, Chicago, IL) and SPSS 12.0 (SPSS).

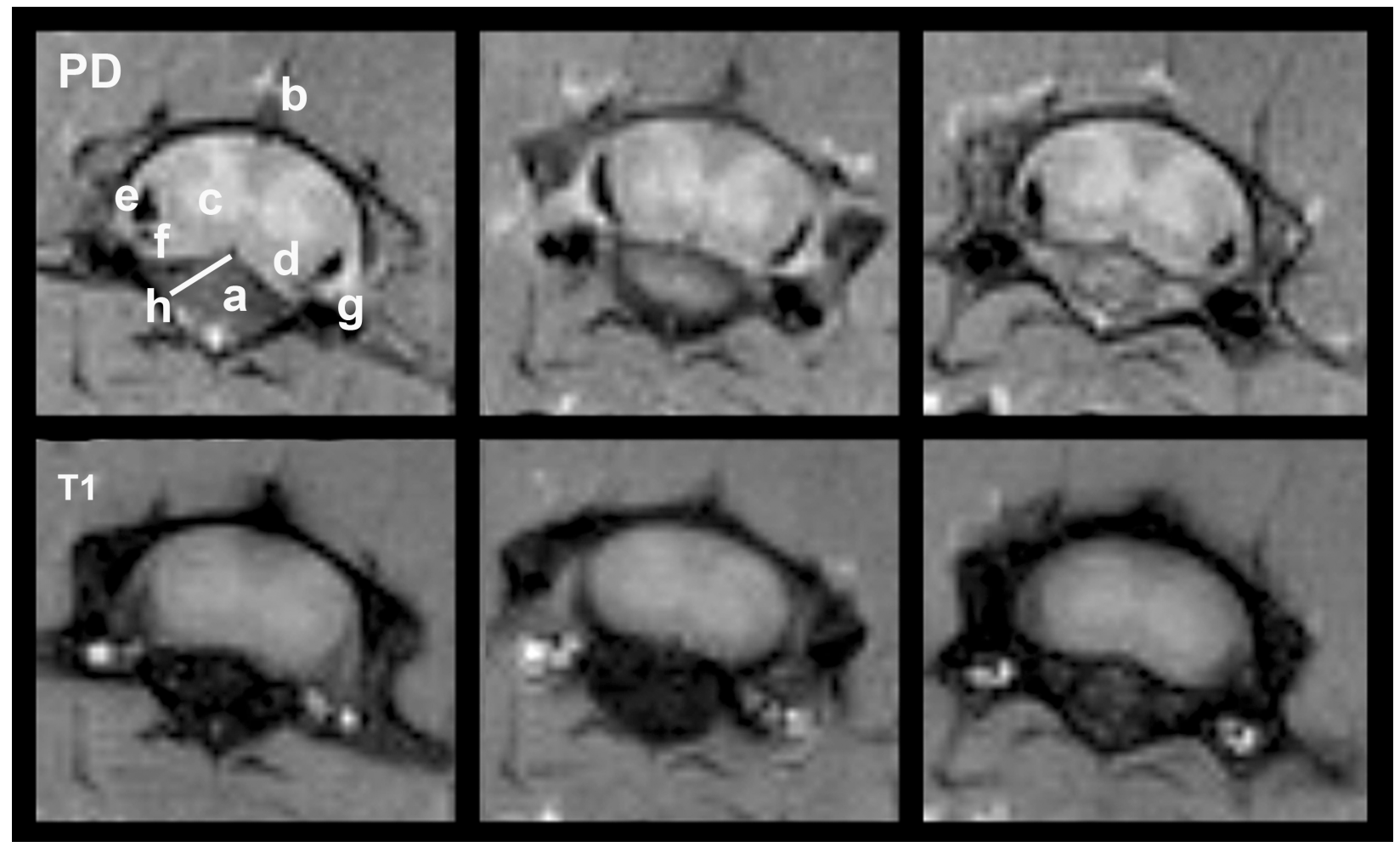

FIG. 1. Consecutive, rostral to caudal (left to right), axial images acquired pre-injury (baseline) at the level of C5 with a resolution of $0.175 \times 0.175 \times 1 \mathrm{~mm}$ and with a 0.1 -mm interslice gap. (Top) PD images. (Bottom) T1 images. In each image: top $=$ dorsal and bottom = ventral. Structures that can be identified include: the vertebral body (a) and spinous process (b), gray (c) and white matter (d), dorsal (e) and ventral roots (f), and ganglia (g). Ventral to the ventral roots, the ventral spinal artery (h) is visible in the ventral midline fissure. 
A

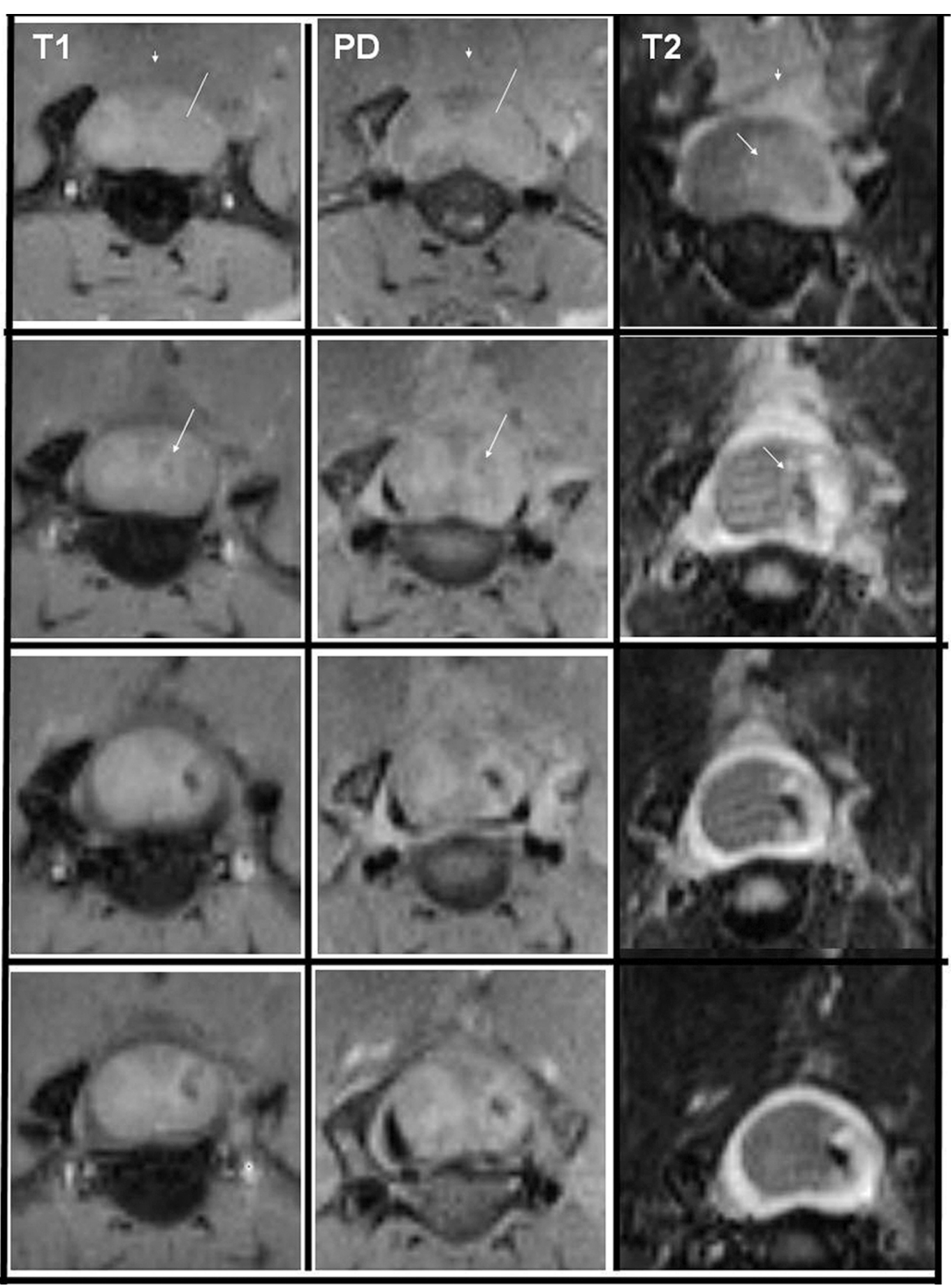

$24 \mathrm{~h}$

7 days

14 days

\section{1 days}

FIG. 2. Development of the lesion center in a $6.25 \mathrm{~g}-\mathrm{cm}(\mathbf{A})$ and a $25 \mathrm{~g}-\mathrm{cm}(\mathbf{B})$ injured rat over time. Representative axial images from the T1 (left), PD (middle), and T2 (right) sequences are shown at $24 \mathrm{~h}$, and at 7, 14, and 21 days after SCI. In each image: top $=$ dorsal and bottom $=$ ventral. Indicated in A are the following: Arrowheads indicate the area of the laminectomy and associated soft tissue edema, noticeable in the T2 images. Lines are directed to the region in the right hemicord where loss of GM-WM differentiation occurs. Long arrows indicate the hypointense area. Short arrows indicate the hyperintense area, seen in the $\mathrm{T} 2$ images. 


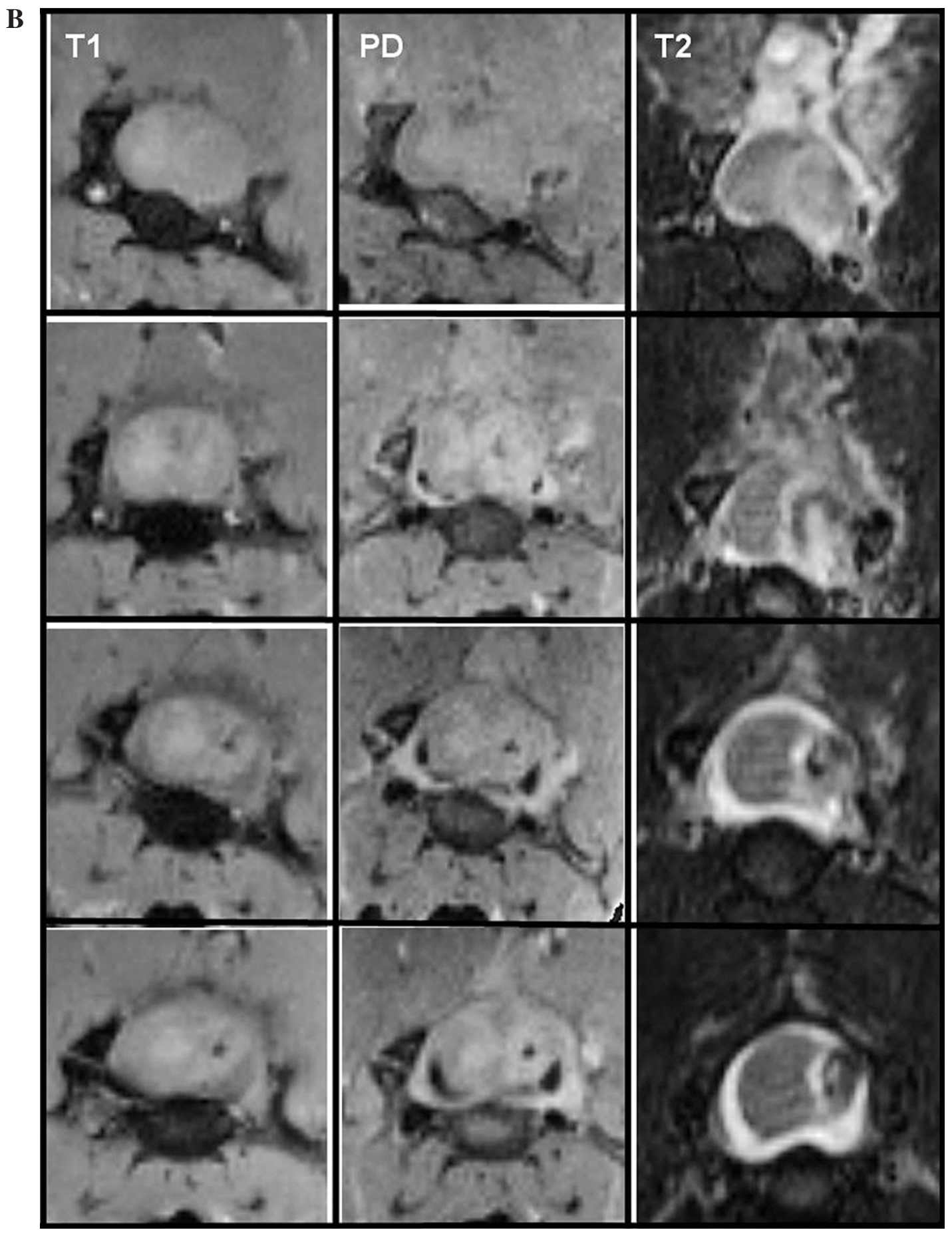

FIG. 2. (Continued).

\section{RESULTS}

Surgical procedures and repeated MRI scans were well tolerated by all animals. One animal in the $25 \mathrm{~g}-\mathrm{cm}$ group was sacrificed early (day 14 after SCI) due to a technical problem with the jugular catheter.

\section{MRI and MRI Quantification}

Examples of pre-injury (baseline) axial MR images of the spinal cord at the level of C5 are shown in Figure 1. These images clearly depict gray matter-white matter (GM-WM) structures and nerve roots, especially on PD 


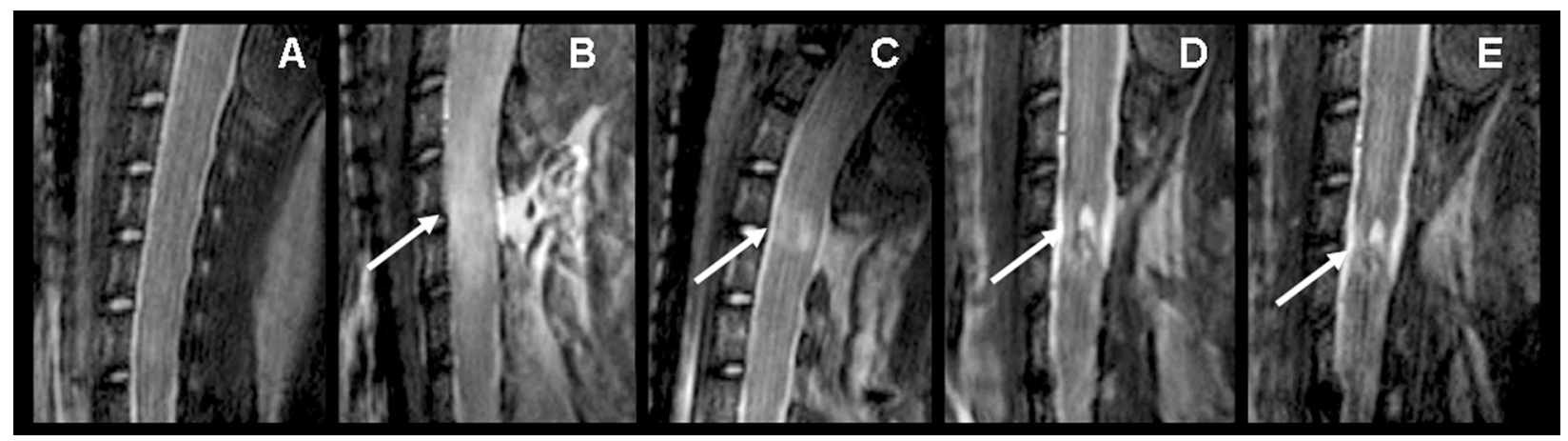

FIG. 3. Sagittal T-2 weighted images (image resolution $182 \times 172 \times 625 \mu \mathrm{m}^{3}$ ) of a $25 \mathrm{~g}$-cm injured rat before injury (A) and at $24 \mathrm{~h}(\mathbf{B}), 7$ days $(\mathbf{C}), 14$ days (D), and 21 days (E) after SCI. The arrows indicate injury location.

weighted images. Components of relevant vasculature and nerve structures can be identified, particularly in T1weighted images. Aside from the clear rim of cerebrospinal fluid surrounding the spinal cord, baseline T2weighted images did not display additional information, and are not shown. After injury, T1 and PD weighted images were considered most useful for assessment of cord swelling, contrast enhancement, and hemorrhage, and T2-weighted images were valuable for assessment of fluid infiltration into the cord, i.e. edema (Figs. 2 and 3).

At $24 \mathrm{~h}$ after injury, significant swelling of the spinal cord, extending rostrocaudally well beyond the level of SCI, and loss of GM-WM differentiation in the right hemicord, were detectable by MRI in both injured groups (Figs. 2A,B and 3). Quantification of hemicord volumes (Fig. 4) demonstrated that in both groups the right and left hemicords were significantly larger at $24 \mathrm{~h}$ after SCI than at all other time points $(p<0.001)$. Furthermore, at $24 \mathrm{~h}$ after SCI, the right hemicord was significantly larger than the left hemicord in the $25 \mathrm{~g}$-cm group $(p<0.001)$, but not in the $6.25 \mathrm{~g}-\mathrm{cm}$ group. Cord swelling at $24 \mathrm{~h}$ after SCI appeared concurrently with the development of hyperintense signal on T2-weighted images (Figs. 2 and $5 \mathrm{~A}-\mathrm{C})$. At the level of the lesion, this hyperintense signal was present in a scattered pattern throughout the majority of the injured hemicord. Moreover, this signal spread to the contralateral side and extended rostrocaudally from the injury epicenter. This phenomenon was seen in all rats that were imaged using this sequence. In Figure 5C, the volume of hyperintense signal is shown over time ( $n=6$ in the 6.25 -mm group and $n=1$ in the 25-mm group). An area of hypointense signal within the

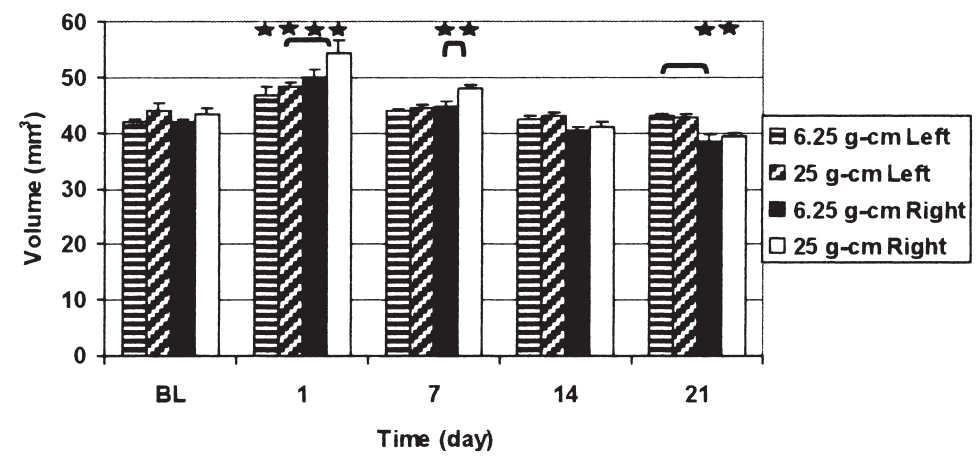

FIG. 4. Left and right hemicord volumes, determined from $\mathrm{T} 1$ and PD weighted images, quantified over a length of $11 \mathrm{~mm}$, shown for both groups of rats. Between-group comparison resulted in a significant difference at 7 days after injury. The volume of the right hemicord of the $25 \mathrm{~g}-\mathrm{cm}$ group was significantly larger than that of the $6.25 \mathrm{~g}$-cm group $(p=0.02$; marked with $\cap$ ). Within-group comparison of left to right hemicord volumes showed a significant difference at day 21 after injury for the $6.25 \mathrm{~g}$ $\mathrm{cm}$ group and at $24 \mathrm{~h}$ after injury for the $25 \mathrm{~g}$-cm group $(p<0.001$; marked with $\cap$ ). Within-group comparison of left and right (lesioned side) hemicords for both groups resulted in the following significant differences $(p<0.001$; marked with $\star)$. The left and right hemicord volumes were significantly larger at $24 \mathrm{~h}$ after SCI than at all other time points. In addition, the right hemicords were significantly larger at 7 days after injury than at baseline and at 14 and 21 days after SCI. Finally, the right hemicords were significantly smaller at 21 days after SCI than at baseline. 

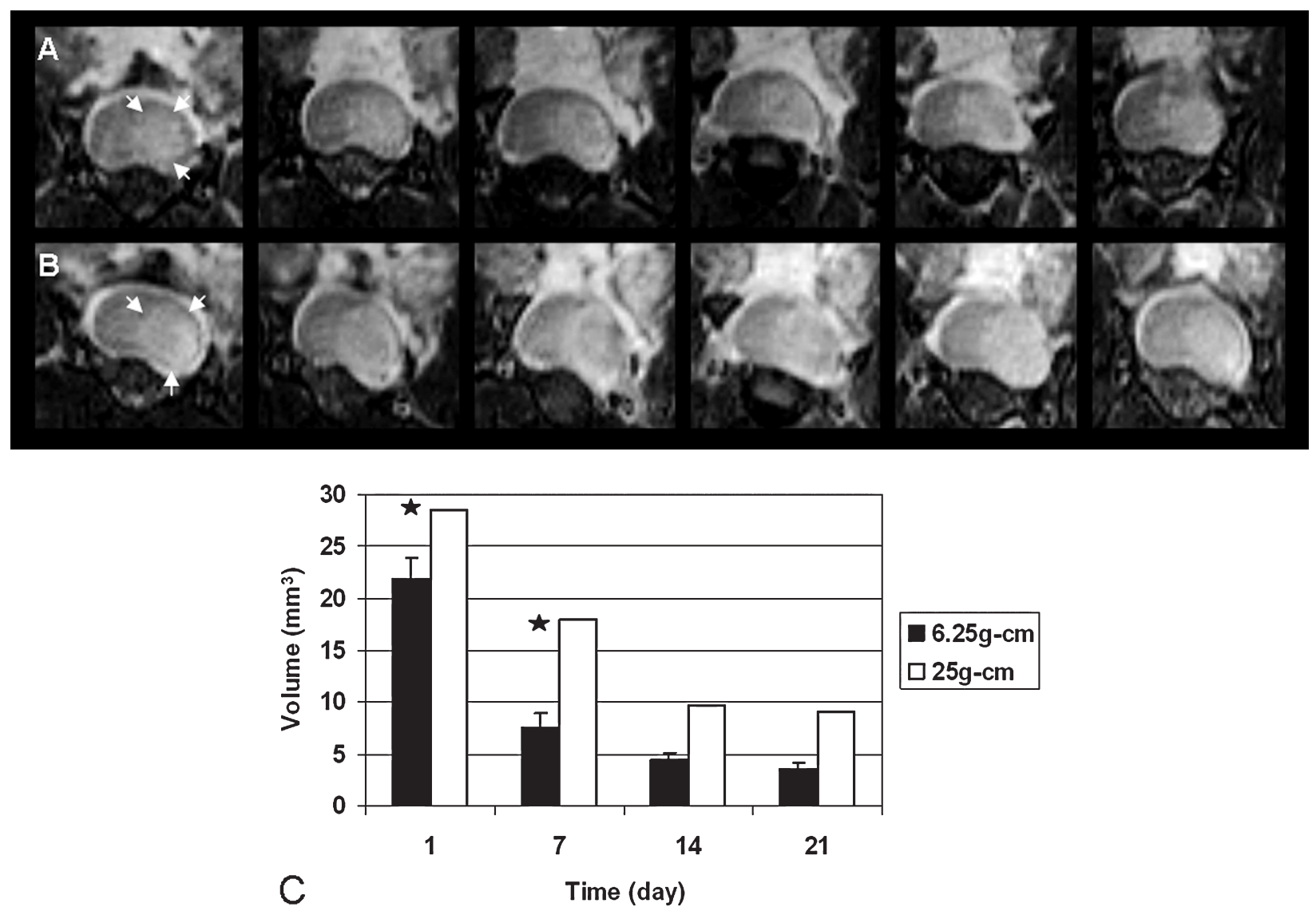

FIG. 5. The rostrocaudal and mediolateral extent of hyperintense signal is shown in a $6.25 \mathrm{~g}-\mathrm{cm}(\mathbf{A})$ and a $25 \mathrm{~g}-\mathrm{cm}(\mathbf{B})$ injured rat at $24 \mathrm{~h}$ after SCI. Representative rostral to caudal consecutive T2-weighted images acquired with a $182 \times 172 \times 625 \mu \mathrm{m}$ resolution are shown. Small arrows in the first images of A and B indicate the rim of hyperintense signal. In each image: top is dorsal and bottom is ventral. (C) The volume of hyperintense signal calculated from the axial T2-weighted images for all $6.25 \mathrm{~g}$-cm injured rats and one $25 \mathrm{~g}-\mathrm{cm}$ injured rat is shown. In the $6.25 \mathrm{~g}-\mathrm{cm}$ group the signal at $24 \mathrm{~h}$ was significantly higher than at all other time points, and the signal on day 7 was significantly higher than on day 21 after SCI $(p<0.001$; marked with $\star)$.

lesioned hemicord at the level of the lesion epicenter was seen in two of the seven animals in the $25-\mathrm{mm}$ group in the T1- and PD-weighted images (images not shown). In addition, at $24 \mathrm{~h}$ after injury, uptake of gadodiamide into the injured spinal cord was seen in T1-weighted images in both groups (Fig. 6).

MR images at 7 days post-injury were characterized by loss of gray matter and development of a hypointense area within the ipsilateral cord (lesioned side) in all animals regardless of injury severity, and this was seen in all types of images (Fig. 2). The localization of this hypointense signal was at the level of the lesion epicenter, and in Figure 7, the volume of this low signal area is shown for both groups over the 3-week study period. Swelling of the cord and volume of hyperintense signal were reduced when compared to $24 \mathrm{~h}$ post-injury (Figs. 4 and 5C), and the GM-WM contrast was restored (Fig. 2). At 7 days post-injury, the ipsilateral cord of the $25 \mathrm{~g}$ - $\mathrm{cm}$ group was significantly larger than the ipsilateral cord of the $6.25 \mathrm{~g}-\mathrm{cm}$ group ( $p=0.02$; Fig. 4). Furthermore, in both groups, the ipsilateral cord was significantly larger at 7 days than at baseline, and at 14 and 21 days after SCI $(p<0.001)$. After gadodiamide administration, obvious signal enhancement was present in the injured hemicord in all $25 \mathrm{~g}-\mathrm{cm}$ injured rats; however, no obvious signal enhancement was seen in the $6.25 \mathrm{~g}-\mathrm{cm}$ injured group. Contrast administration was subsequently discontinued in the latter group.

At 14 and 21 days post-injury, MR images showed further reduction of swelling and hyperintense signal (Figs. 2,4 , and 5C), and further development of the area of hypointense signal (Fig. 7). The appearance of hyperintense signal appeared to change from a scattered pattern throughout the lesioned spinal cord to a more organized rim of hyperintensity located around the hypointense area (Fig. 2). At 14 days after SCI, there was no obvious sig- 


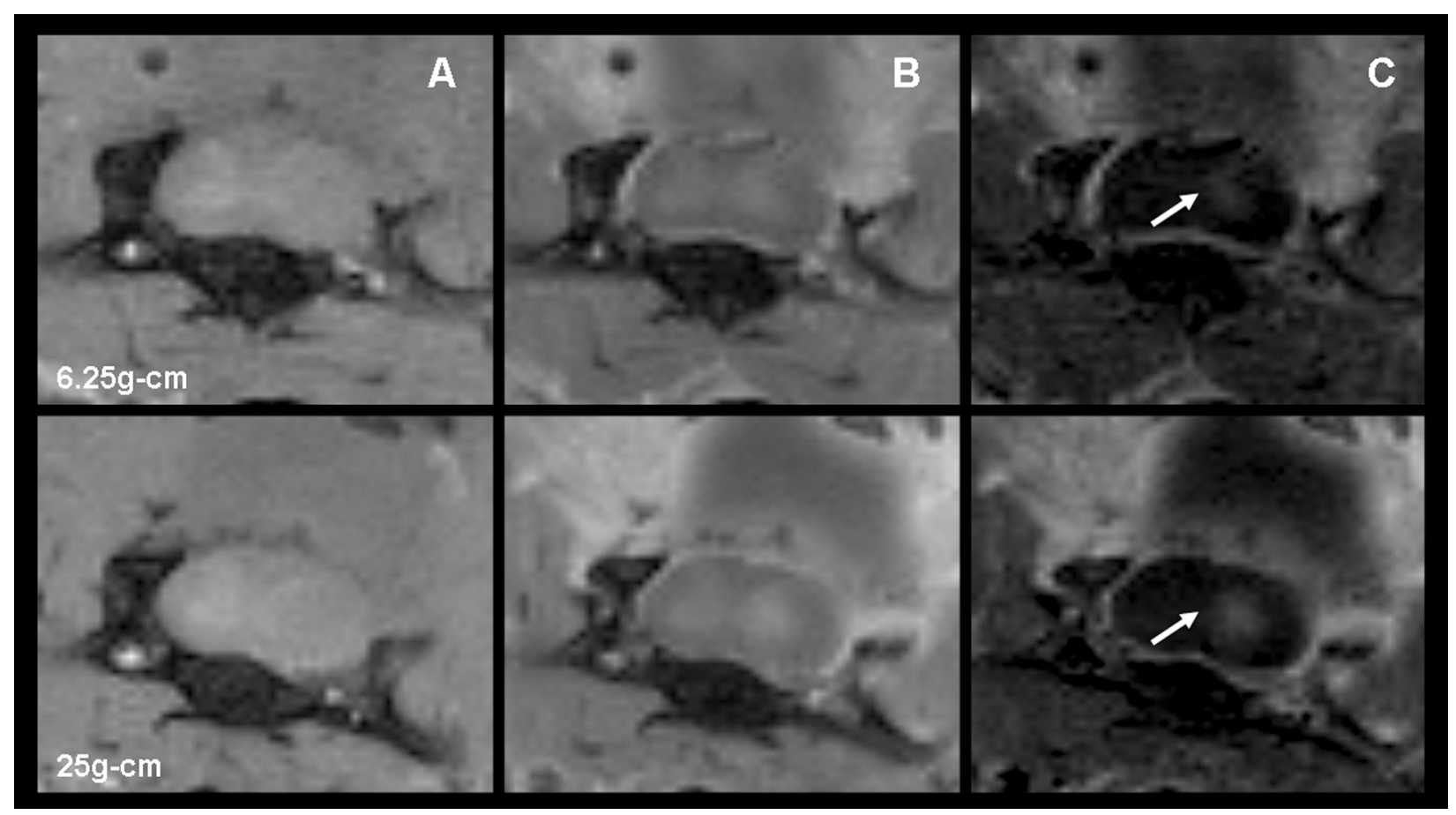

FIG. 6. T1 weighted images of a $6.25 \mathrm{~g}-\mathrm{cm}$ (upper row) and a $25 \mathrm{~g}-\mathrm{cm}$ (lower row) injured rat acquired before (A) and after (B) Ominiscan administration at $24 \mathrm{~h}$ post-SCI. The enhancement of the injured spinal cord, indicative of BSCB leakage is seen in the difference image (C), resulting from subtraction of post-contrast (B) from pre-contrast image (A). For the $25 \mathrm{~g}$-cm injured rat, the region of enhancement is larger than the one observed for the $6.25 \mathrm{~g}-\mathrm{cm}$ injured rat (arrows).

nal enhancement after gadodiamide administration in the T1-weighted images of the $25 \mathrm{~g}-\mathrm{cm}$ injured rats. These last in vivo MR images also demonstrated collapse of the injured hemicord (Fig. 2) which was confirmed by quantification of the hemicord volumes that demonstrated a significantly smaller right sided hemicord compared to the left side in both groups at 21 days after SCI $(p<$ 0.001; Fig. 4). Furthermore, the volume of hypointense signal throughout the lesion cavity was significantly larger in the $25 \mathrm{~g}-\mathrm{cm}$ compared to the $6.25 \mathrm{~g}-\mathrm{cm}$ group at both 14 and 21 days after SCI ( $p<0.04$; Fig. 7). The area of hypointense signal at the lesion epicenter deter-

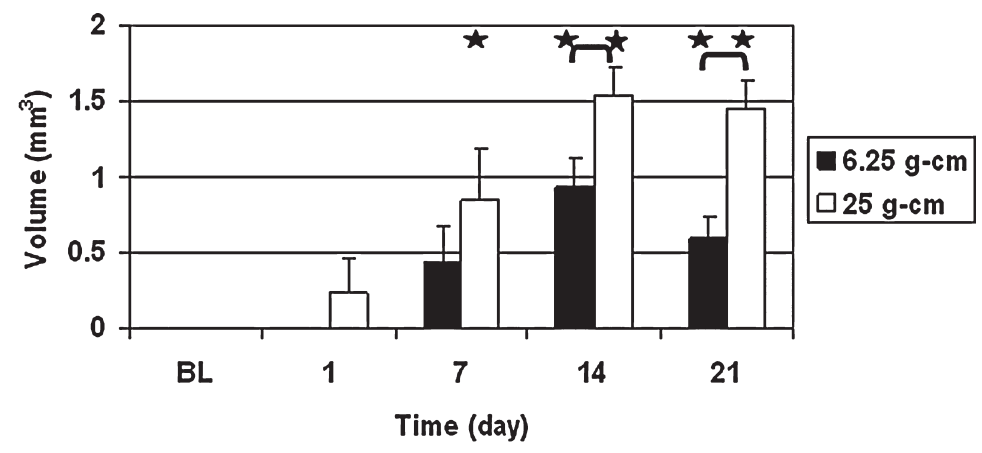

FIG. 7. Volume of hypointense signal for both groups of injured rats determined from T1 and PD weighted images. Betweengroup comparison demonstrated a significantly larger volume in the $25 \mathrm{~g}-\mathrm{cm}$ group than in the $6.25 \mathrm{~g}-\mathrm{cm}$ group at 14 and 21 days after SCI $(p<0.04$, marked with $\cap$ ). Within-group comparison showed that in the 6.25 group the volume of hypointense signal was significantly larger at 14 and 21 days after SCI compared to baseline (BL) and $24 \mathrm{~h}$ after SCI $(p<0.001$; marked with $\star)$. Furthermore, in the $25 \mathrm{~g}-\mathrm{cm}$ group, this volume was significantly larger at 14 and 21 days after injury compared to baseline and $24 \mathrm{~h}$ after SCI $(p<0.001$; marked with $\star)$, and at 7 days after SCI this volume was significantly larger than at baseline $(p<$ 0.001 ; marked with $\star$ ). 
mined 21 days after SCI in vivo was significantly smaller in the $6.25 \mathrm{~g}-\mathrm{cm}$ group when compared to the $25 \mathrm{~g}-\mathrm{cm}$ group ( $p<0.008$; see Fig. 11B below).

\section{Behavior}

Unilateral SCI resulted in a BBB score of 11 in all animals. There was no change in BBB score throughout the study period. The BBB score is predominantly a measure of hind limb function and in this case indicates that, when the rat is walking, there is consistent weight supported stepping but no coordination between hind and front limbs; this is understood to be primarily a forelimb deficit, and is not really comparable to BBB scores from thoracic-injured rats. Differences in hind limb function were seen, however, using the BBB subscore (Popovich et al., 1999). Figure $8 \mathrm{~A}$ shows that, at $48 \mathrm{~h}$ and on day 22 after SCI, the BBB subscores for animals in the $6.25 \mathrm{~g}$ $\mathrm{cm}$ group ( $8.8 \pm 0.5$ and $11.3 \pm 0.2$, respectively) were significantly higher than the subscores for animals in the $25 \mathrm{~g}$-cm group $(5.4 \pm 0.8$ and $10 \pm 0.4$, respectively; $p<$ $0.05)$, indicating that fewer hind limb placement and tail carriage mistakes were made.

After SCI, right front limb function was significantly impaired in all animals, which is evident from the grooming and paw preference tests (Fig. 8B,C). Figure 8B shows that grooming behavior of the animals in the $6.25 \mathrm{~g}-\mathrm{cm}$ group recovered faster and to a significantly higher degree than that of the animals in the $25 \mathrm{~g}-\mathrm{cm}$ group. Differences in performance in the grooming test were significant on days 8,15 , and 22 after SCI $(p<$ 0.001 ). The paw preference test demonstrated that at 22 days after SCI, the animals in the $25 \mathrm{~g}$-cm group rarely used their right front limb during exploratory behavior (Fig. 8C). Consequently, these animals used their left front paw alone in $99.4 \pm 0.4 \%$ of placements. In the $6.25 \mathrm{~g}-\mathrm{cm}$ group, animals used their right front limb significantly more than the animals in the $25 \mathrm{~g}-\mathrm{cm}$ group $(p=0.015)$; use of the left front limb alone occurred in $83 \pm 8 \%$ of placements.

\section{Post-Mortem Imaging}

MRI of the ex vivo in situ spinal cord and excised cords embedded in agar, clearly showed a central hypointense area surrounded by a hyperintense area, as well as collapse of the ipsilateral side of the spinal cord (Fig. 9A,B). Figure 10A shows the lesion epicenters, recreated from histopathology, of the individual animals in each group. Sparing of white and gray matter in the right hemicord was seen in MR images (in vivo, ex vivo in situ, and agarembedded cords) and was confirmed by histopathology (Figs. 9 and 10A,B). More ipsilateral and contralateral damage at injury epicenters was present in the $25 \mathrm{~g}-\mathrm{cm}$ injured rats. Ipsilateral white matter sparing was significantly larger in the $6.25 \mathrm{~g}-\mathrm{cm}$ group when compared to the $25 \mathrm{~g}$-cm group ( $p=0.015$; Fig. 10B). In both groups no motor neurons were seen in the injured hemicord at the level of lesion epicenter. More motor neurons were counted in the $6.25 \mathrm{~g}-\mathrm{cm}$ group when compared to the $25 \mathrm{~g}-\mathrm{cm}$ group in the contralateral (uninjured) hemicord $(p<0.001$; Fig. 10C).

In Figure 11A, the lesion length, as determined at 3 weeks after injury by in vivo MRI and post-mortem using three imaging modalities-MRI in situ, MRI of agarembedded excised cords, and microscopy-is presented for both groups. All imaging modalities demonstrated a significant difference in lesion length between the two injured groups $(p<0.004)$. There were no significant differences between measurements made by using the different imaging methods.

The area of the lesion cavity at the injury epicenter, determined from the hypointense area seen in the in vivo and post mortem MR images and from histopathologic examination (Figs. 9 and 10A), is shown in Figure 11B. In vivo $\mathrm{MR}$ images at 21 days after $\mathrm{SCI}$ demonstrated a
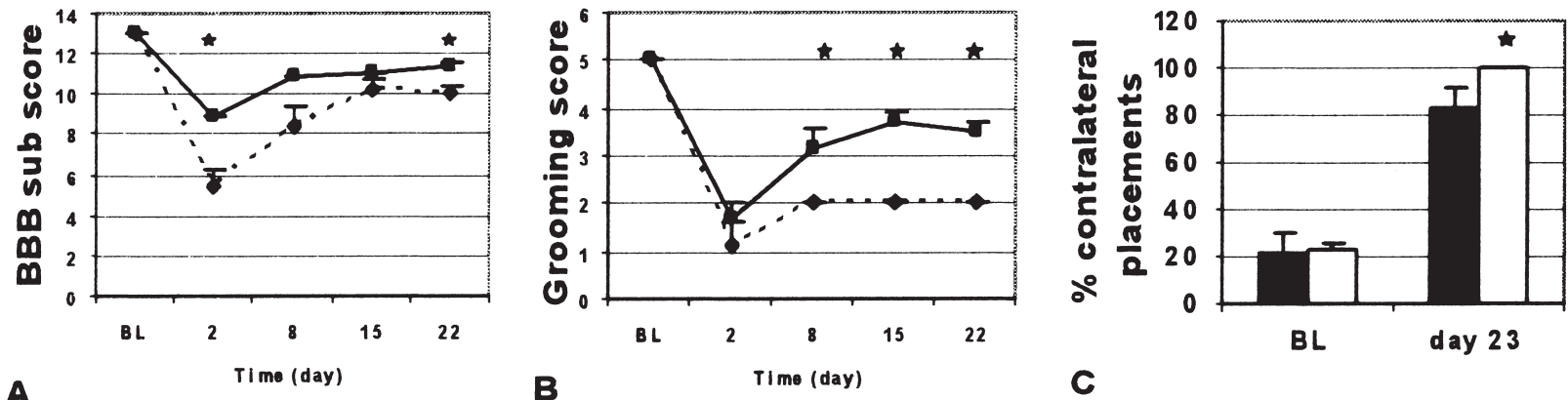

FIG. 8. Neurobehavioral test results: BBB subscore (A), grooming test $(\mathbf{B})$, and paw preference test $(\mathbf{C})$. The $6.25 \mathrm{~g}$-cm group is indicated with the closed line (A, B) and black bar $(\mathbf{C})$. The $25 \mathrm{~g}-\mathrm{cm}$ group is indicated by the dashed line (A, B) and white bar (C). Significant differences between the groups are indicated with $\star$. $p<0.05$ (A), $p<0.001$ (B), and $p=0.015$ (C). 


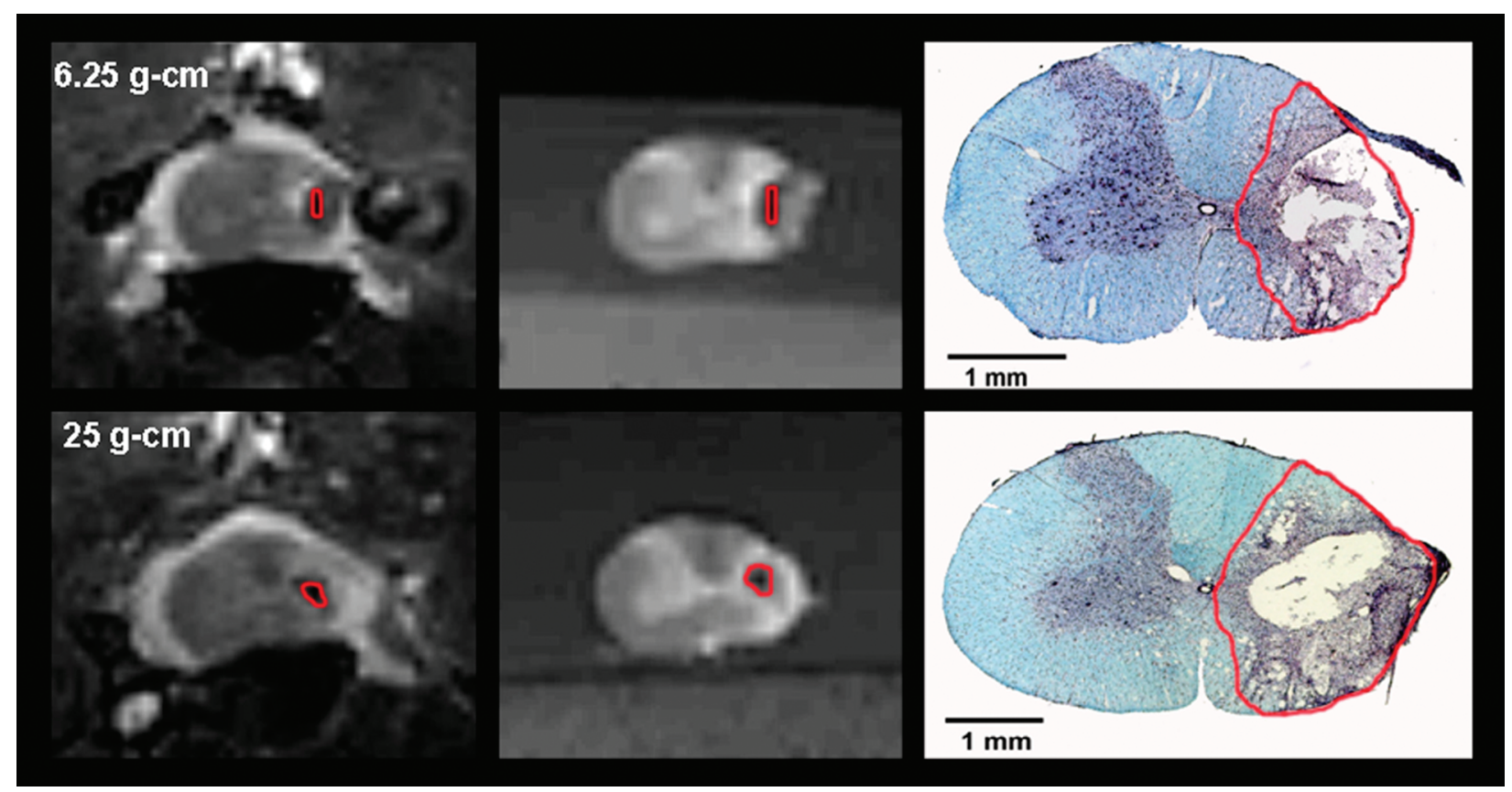

FIG. 9. Post-mortem images of the lesion epicenter from one representative animal from the $6.25 \mathrm{~g}-\mathrm{cm}$ (top) and $25 \mathrm{~g}-\mathrm{cm}$ (bottom) injury groups. Shown are MRI post-mortem in situ (left), MRI post-mortem after embedding the spinal cord in agar (middle), and a 20- $\mu \mathrm{m}$ section imaged using microscopy (right). The red line indicates the areas determined to be the hypointense or lesioned area in MRI and histopathology sections, respectively. In each image, top is dorsal and bottom is ventral.

significantly smaller hypointense area in the $6.25 \mathrm{~g}-\mathrm{cm}$ group when compared to the $25 \mathrm{~g}-\mathrm{cm}$ group $(p<0.008)$. However, none of the other imaging modalities demonstrated this significant difference. The lesion cavity areas determined by histopathology were significantly larger than those determined by MRI (in situ and agar) in both groups ( $p<0.001$ in the $6.25 \mathrm{~g}$-cm group; $p<0.004$ in the $25 \mathrm{~g}-\mathrm{cm}$ group).

\section{Correlation between MRI, Behavior, and Histopathology}

Significant correlations exist between cord swelling at 7 days after SCI and histological measures such as lesion length and gray and white matter sparing (Table 1). Other in vivo MRI parameters that were highly correlated with histopathological outcomes included quantity of hyperintense signal at all time points, hypointense signal at 21 days, and lesion length at 21 days after SCI. Furthermore, quantification of hypointense signal and lesion length at 21 days after SCI determined by in vivo MRI was highly correlated to neurobehavioral outcomes, in particular to the BBB subscore and the grooming test.

Significant correlations also exist between the different parameters determined by MRI in vivo (Table 2). Swelling at $24 \mathrm{~h}$ after SCI is highly correlated to quantity of hypointense signal at 21 days after SCI. In addi- tion, hyperintense signal at $24 \mathrm{~h}$ after SCI is highly correlated to swelling at 7 days after SCI, and swelling at 7 days after SCI is highly correlated to hyperintense signal at 21 days after SCI. Furthermore, hyperintense signal at 7,14 , and 21 days after SCI is highly correlated to lesion length at 21 days after SCI. Finally, hypointense signal and lesion length determined at 21 days after SCI are highly correlated.

\section{DISCUSSION}

MRI is a valuable imaging modality to assess temporal evolution of SCI and to distinguish different severities of cervical SCI in rats. In our study, quantification of cord swelling, hypointense and hyperintense signal, and lesion length from MR images appeared to be the most valuable parameters to determine since these parameters were highly correlated to behavioral outcomes and histopathological characteristics of the lesion. This corresponds to MRI findings in humans with SCI; extent of hemorrhage and edema appear predictive of and/or provide accurate prognostic information about neurologic outcome (Boldin et al., 2006; Miyanji et al, 2007; Selden et al., 1999).

During the course of this study, we imaged 12 rats five consecutive times in vivo, using a non-invasive MRI tech- 


\section{A $6.25 \mathrm{~g} \mathrm{~cm}$}

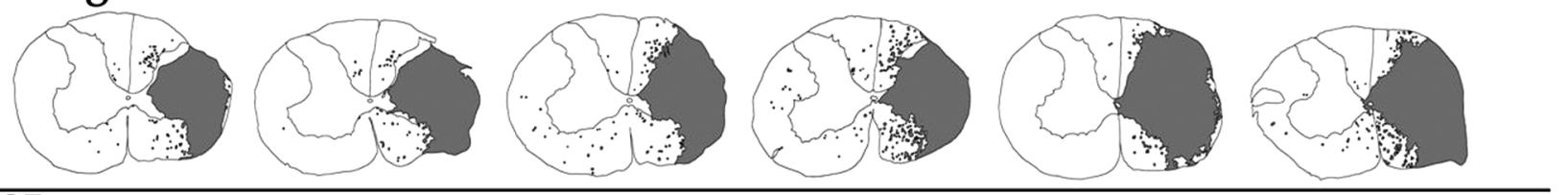

\section{$25 \mathrm{~g} \mathrm{~cm}$}
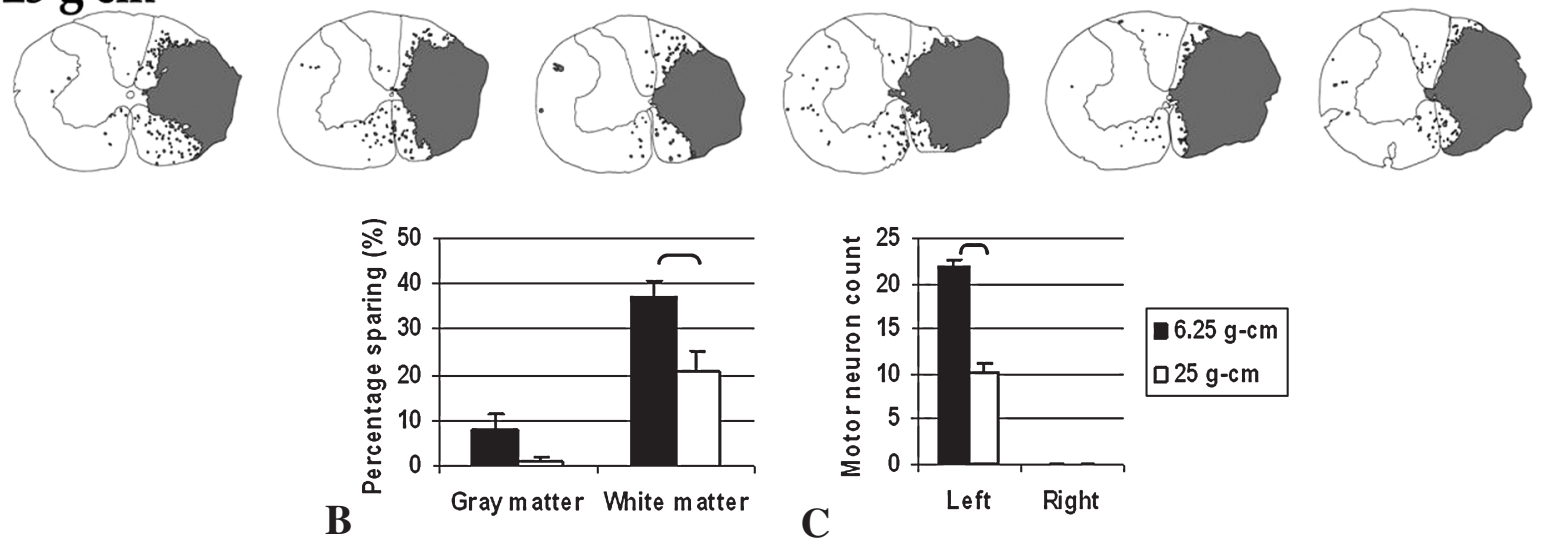

FIG. 10. Histopathology: representations of the individual lesion epicenters (gray area) of individual animals of the $6.25 \mathrm{~g}-\mathrm{cm}$ and $25 \mathrm{~g}-\mathrm{cm}$ groups $(\mathbf{A})$, percentage gray and white matter sparing $(\mathbf{B})$, and motor neuron counts $(\mathbf{C})$. Images in A show that there is typically more ipsilateral gray and white matter sparing in the $6.25 \mathrm{~g}-\mathrm{cm}$ group when compared to the $25 \mathrm{~g}$-cm group and more contralateral white matter damage in the $25 \mathrm{~g}-\mathrm{cm}$ group when compared to the $6.25 \mathrm{~g}-\mathrm{cm}$ group. Furthermore, these images show overlap between the two groups with regards to extent of the lesion at epicenter. In each image, top is dorsal and bottom is ventral. In B, sparing of gray and white matter at injury epicenter, expressed as percentage from contralateral gray and white matter, is shown determined by histology. White matter sparing was significantly higher in the $6.25 \mathrm{~g}$-cm group when compared to the $25 \mathrm{~g}-\mathrm{cm}$ group $(p=0.015$; marked with $\cap$ ). Significantly more large motor neurons were counted in the contralateral (uninjured) hemicord at the level of lesion epicenter of animals in the $6.25 \mathrm{~g}$-cm group than of animals in the $25 \mathrm{~g}$-cm group (C; $p<$ 0.001 ; marked with $\cap$ ).

nique using surface, rather than implanted, coils. MRI depicted significant differences between the $6.25 \mathrm{~g}-\mathrm{cm}$ and $25 \mathrm{~g}-\mathrm{cm}$ hemicontusion injury with regards to swelling of the spinal cord and contrast enhancement of the lesion in the acute phase of injury. In addition, in the subacute to chronic phase ( $>7$ days after SCI) MRI demonstrated significant differences between the two groups with regards to lesion length and volume. SCI results in loss of normal cord cytoarchitecture characterized by MRI in the immediate phase by loss of GM-WM differentiation, evidence of hemorrhage and accumulation of edema (represented by hypointense signal in T1-weighted images
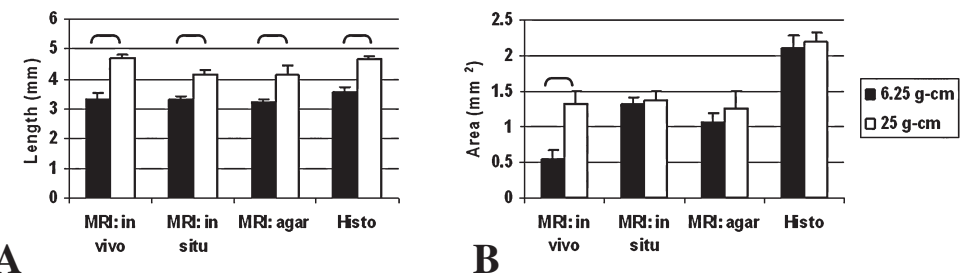

FIG. 11. MRI versus histology: Lesion length determined by MRI in vivo and post mortem by MRI in situ (left), and of agarembedded cords (middle), and by microscopy (right; A). All imaging modalities demonstrated a significant difference between the two injured groups $(p<0.004$; marked with $\cap$ ). (B) The area of the lesion at injury epicenter is shown determined by MRI in vivo and post mortem by MRI in situ (left), and of agar-embedded cords (middle), and by microscopy (right). The area of the lesion was determined from the hypointense area in the MR images and by histopathological verification of gray and white matter damage using microscopy. A significant difference between the two groups was identified only with MRI in vivo ( $p<0.008$; marked with $\cap$ ). 
MIHAI ET AL.

Table 1. Correlation between MRI and Behavioral and Histopathological Outcomes ( $r$-Values)

\begin{tabular}{|c|c|c|c|c|c|c|}
\hline \multirow[b]{2}{*}{$M R I$ in vivo } & \multicolumn{2}{|c|}{ Behavior $22 d$} & \multicolumn{4}{|c|}{ Histology } \\
\hline & $B B B$ subscore & Grooming & Length & WM sparing & GM sparing & Motor neurons \\
\hline Swelling 7d & -0.3 & -0.3 & $0.6^{*}$ & $-0.8 * * *$ & $-0.7 * *$ & -0.4 \\
\hline Hyperintensity $24 \mathrm{~h}$ & $-0.9 * *$ & -0.2 & $0.8^{*}$ & $-0.9 * * *$ & $-0.9 * * *$ & -0.4 \\
\hline Hyperintensity 7d & $-0.9 * * *$ & -0.6 & $0.9 * * *$ & $-0.98 * * *$ & $-0.8 *$ & -0.6 \\
\hline Hyperintensity $14 d$ & $-0.8 * *$ & -0.5 & $0.9 * * *$ & $-0.95 * * *$ & -0.7 & -0.6 \\
\hline Hyperintensity $21 \mathrm{~d}$ & $-0.96^{* * * *}$ & -0.6 & $0.9 * * *$ & $-0.98 * * *$ & -0.8 & -0.8 \\
\hline Hypointense region $21 \mathrm{D}$ & $-0.7 *$ & $-0.6^{*}$ & $0.6^{*}$ & -0.5 & -0.4 & $-0.6^{*}$ \\
\hline Length $21 \mathrm{~d}$ & $-0.7^{*}$ & $-0.7 * * *$ & $0.95 * * *$ & $-0.8 * * *$ & $-0.7 * * *$ & $-0.8 * * *$ \\
\hline
\end{tabular}

Significance: $* p \leq 0.05, * * p \leq 0.02, * * * p \leq 0.01$.

Note that the volumetric hyperintensity data ia acquired from six animals in the $6.25 \mathrm{~g}-\mathrm{cm}$ group and one animal from the $25 \mathrm{~g}-\mathrm{cm}$ group.

MRI, magnetic resonance imaging; d, days,; BBB, Basso, Beattie, and Bresnahan scale; WM, white matter; GM, gray matter.

and hyperintense signal in T2-weighted images), cord swelling (represented by increase in cord volume), and blood spinal cord barrier (BSCB) disruption, represented by extravasation of contrast agent into the spinal cord. Measurements of swelling, such as cord size and derived volumes, are easily obtained from MR images, and, from our results, appear to be correlated to injury severity. Cord swelling occurs because of disruption of vasculature and alteration of local fluid compartmentalization, with subsequent accumulation of blood and edema in and around the site of the contusion injury. Blood and/or blood products are recognized by a hypointense signal in T2-weighted images, since $\mathrm{Fe}^{2+}$ deoxyhemoglobin and
$\mathrm{Fe}^{3+}$ methemoglobin shorten the $\mathrm{T} 2$ relaxation times (Duncan et al., 1992; Weirich et al., 1990). Evidence of presumed hemorrhage was visible in a subset of the severely injured animals in our study at $24 \mathrm{~h}$, and in all of our animals, at 7 days after injury. Hemorrhage is primarily localized in the lesion center gray matter, and, in our hemicontusion injury, this central hypointense core was thus seen ipsilaterally. Hemorrhage was seen with concomitant loss of GM-WM differentiation and accumulation of extravascular fluid, similar to that shown by other groups (Bilgen et al., 2000; Duncan et al., 1992; Falconer et al., 1994; Hackney et al., 1994; Narayana et al., 2004; Ohta et al., 1999; Weber et al., 2006).

Table 2. MRI In Vivo: Correlation between Quantified Parameters ( $r$-Values)

\begin{tabular}{|c|c|c|c|c|c|c|c|c|}
\hline & \multicolumn{2}{|c|}{ Swelling } & \multicolumn{4}{|c|}{$\begin{array}{l}\text { Hyperintense } \\
\text { region }\end{array}$} & \multirow{2}{*}{$\begin{array}{c}\begin{array}{c}\text { Hypointense } \\
\text { region }\end{array} \\
21 d\end{array}$} & \multirow{2}{*}{$\frac{\text { Length }}{21 d}$} \\
\hline & $24 h$ & $7 d$ & $24 h$ & $7 d$ & $14 d$ & $21 d$ & & \\
\hline \multicolumn{9}{|c|}{ Swelling } \\
\hline $24 \mathrm{~h}$ & & 0.4 & 0.3 & 0.2 & 0.3 & 0.4 & $0.7 *$ & 0.2 \\
\hline $7 \mathrm{~d}$ & 0.4 & & $0.9 * * *$ & $0.8^{*}$ & 0.7 & $0.9 * *$ & 0.5 & 0.5 \\
\hline \multicolumn{9}{|c|}{ Hyperintense region } \\
\hline $24 \mathrm{~h}$ & 0.3 & $0.9 * * *$ & & $0.8^{*}$ & 0.7 & $0.8 *$ & 0.2 & 0.5 \\
\hline $7 d$ & 0.2 & $0.8^{*}$ & $0.8^{*}$ & & $0.98 * * *$ & $0.99 * * *$ & 0.5 & $0.8 * *$ \\
\hline $14 d$ & 0.3 & 0.7 & 0.7 & $0.98 * * *$ & & $0.96^{* * *}$ & 0.5 & $0.8 * *$ \\
\hline $21 d$ & 0.4 & $0.9 * *$ & $0.8^{*}$ & $0.99 * * *$ & $0.96 * * *$ & & 0.6 & $0.9 * *$ \\
\hline \multicolumn{9}{|c|}{ Hypointense region } \\
\hline $21 \mathrm{~d}$ & $0.7 *$ & 0.5 & 0.2 & 0.5 & 0.5 & 0.6 & & $0.6^{*}$ \\
\hline \multicolumn{9}{|l|}{ Length } \\
\hline $21 \mathrm{~d}$ & 0.2 & 0.5 & 0.5 & $0.8 * *$ & $0.8 * *$ & $0.9 * *$ & $0.6^{*}$ & \\
\hline
\end{tabular}

Significance: $* p \leq 0.5, * * p \leq 0.02, * * * p \leq 0.01$.

Note that the volumetric hyperintensity data ia acquired from six animals in the $6.25 \mathrm{~g}-\mathrm{cm}$ group and one animal from the $25 \mathrm{~g}$-cm group.

MRI, magnetic resonance imaging; d, days; h, hours. 


\section{MRI IN LONGITUDINAL COMPARISON OF UNILATERAL CERVICAL SPINAL CORD INJURIES}

The scattered hyperintense signal on T2-weighted images during the acute phase after SCI has been shown to represent edema (Bilgen et al., 2000; Narayana et al., 1999; Ohta et al., 1999). Furthermore, Narayana et al. (1999) suggest the accumulation of edema in the white matter is also responsible for the loss of GM-WM differentiation that is seen immediately after injury. In our study we show that accumulation of edema occurred concurrently with the development of cord swelling. Edema was seen at the level of SCI, and surrounding the lesion site with spread occurring in both mediolateral and rostrocaudal directions. Gradual resolution of the acute edema occurred over a 2 -week period during which the appearance of the scattered hyperintense pattern changed into a hyperintense rim surrounding the hypointense core of blood (products). Our findings confirm a causal relationship between edema and swelling, since volume increase occurs simultaneously with appearance of hyperintense voxels on T2-weighted images. Furthermore, both edema and cord swelling was present in both left and right sides of the spinal cord. We show a high correlation between cord swelling and quantity of hyperintense signal. Moreover, reduction of hyperintense signal during the first week after SCI occurred simultaneously with reduction of cord swelling. As far as the authors are aware, this represents a finding that has not been reported in a quantitative fashion before.

Another process that occurs after SCI that can be assessed by MRI is disruption of the BSCB. Damage to this barrier directly affects pathophysiological processes of lesion development and penetration of potential therapeutic agents from the systemic circulation into the central nervous system. Although MRI characteristics of BSCB disruption after SCI have been extensively studied by others (Berens et al., 2005; Bilgen et al., 2002; Bilgen and Narayana, 2001; Runge et al., 1997; Schwartz, 2005; Whetstone et al., 2003), no longitudinal studies have compared integrity of BSCB between two injury severities. Our results indicate a significant difference between the two study groups at 7 days post-injury, with contrast enhancement of the lesion being much stronger in the severely injured animals than in the mildly injured ones. This is in agreement with Bilgen et al. (2002), who showed a strong correlation between the volume of contrast enhancement and results of neurobehavioral tests in a complex study using dynamic changes in contrast enhancement and a statistical detection algorithm. Although we were able to demonstrate a qualitative difference in BSCB permeability between our two study groups using MRI, we cannot specifically comment about duration of BSCB disruption. Molecular tracing studies and MRI studies have indicated that permeability of the BSCB is increased for at least 30-42 days after SCI (Bilgen et al., 2001a; Popovich et al., 1996), so, although we did not see obvious penetration of contrast agent into the spinal cord at later time points, this most likely suggests partial restitution of barrier function, rather than full BSCB restoration. Our results indicate that damage to the BSCB is more pronounced in the severely injured group and that restoration of BSCB is delayed in this group when compared to the mildly injured animals.

It is noteworthy that most significant differences during the acute phase of SCI between the two injury groups were identified at 7 days after injury, which suggests that the pathophysiological processes that occur during this acute phase of SCI, including accumulation of edema, BSCB disruption, and cord swelling are interrelated. Also, this demonstrates that differences in some of these injury severity related processes do not become apparent until the lesion has progressed over time. Furthermore, around that time, during the first 2 weeks after injury, most improvement is seen with regards to behavioral outcomes. We propose that one of the reasons this might occur, is that cord swelling and edema resolve and the BSCB is, at least partially, restored. Interestingly the phenomenon of improved behavior occurs despite ongoing secondary injury and development of a lesion cavity.

The BBB subscore analysis and assessment of grooming were the most informative neurobehavioral tests in this study. Both of these tests quantify the acute debilitation that occurs after SCI and the subsequent recovery of function that occurs 2-15 days after injury. After 15 days post-SCI, little to no further improvement of function was observed with either of the tests. Both tests correlated highly to contusion parameters and MRI observations. There was no overlap between the $6.25 \mathrm{~g}-\mathrm{cm}$ and $25 \mathrm{~g}-\mathrm{cm}$ groups with regards to behavioral analyses. When comparing behavior and histopathological data from our study with that of Gensel et al. (2006), we see that the animals in our $6.25 \mathrm{~g}-\mathrm{cm}$ group on average had more severe injuries than Gensel et al.'s $6.25 \mathrm{~g}$-cm group, which was evident both from behavioral scores and from ipsilateral tissue sparing seen on histopathology. Withingroup variability is typically more pronounced in milder lesions, and may account for the slightly more severe injury seen in our $6.25 \mathrm{~g}-\mathrm{cm}$ animals. There was less variability in our $25 \mathrm{~g}-\mathrm{cm}$ group. As expected, behavior was worse in our $25 \mathrm{~g}-\mathrm{cm}$ group compared to the $12.5 \mathrm{~g}-\mathrm{cm}$ group in the study by Gensel et al. (2006). However, the lesion areas from histopathology drawings and tissue sparing at the level of lesion epicenter appear very similar between these two groups. This may be due to the biomechanical properties of this type of lesion that perhaps at increased severity shows more rostrocaudal than mediolateral spread. Similar to Gensel et al. (2006) we 


\section{MIHAI ET AL.}

determined near complete loss of ipsilateral gray matter at injury epicenter for both groups.

Evolution of pathology in both injury groups in our study was consistent with previously published studies that describe the evolution of SCI in rats using in vivo or ex vivo MRI with the main goal to investigate the spatiotemporal MRI characteristics of SCI and to compare these to histopathology. Older reports were performed at lower field strengths between 1.5-2T with or without implantable RF coils. These studies examined acute changes in vivo in one lesion severity (Bilgen and Narayana, 2001; Duncan et al., 1992) and temporal changes ex vivo (Hackney et al., 1994) or in vivo (Bilgen et al., 2001a, 2002; Falconer et al., 1994; Narayana et al., 1999; Runge et al., 1997) in thoracic SCI. Just one study used in vivo MRI to examine two cervical lesion severities imaged at two time points and compared that with histopathology and behavioral tests (Ohta et al., 1999). With technological advancement, most recent in vivo MRI studies have been performed using field strengths of 4.7-7 T. These studies have either used further advanced imaging strategies such as diffusion tensor (Deo et al., 2006) and/or have used implantable RF coils (Bilgen et al., 2001b), or evaluated temporal changes of just one injury severity (Narayana et al., 2004). In one study performed at 17.6 $\mathrm{T}$, high quality in vivo images were acquired; however, animal survival during MRI was poor (Weber et al., 2006). To date, our study seems the most comprehensive, with acute and chronic repetitive in vivo MR images from the same animals, ex vivo images, histopathology, and behavioral analysis available for two severities of a cervical hemicontusion SCI. Furthermore, we obtained our MRI data using non-invasive standard imaging techniques that did not require animal intubation, surgical implantation of RF coils, or extremely high field strength.

Similar to the findings from previous studies, in our study MRI showed presence of the hypointense core and development of a hyperintense ring around the core during the subacute to chronic phase of SCI. Histopathology in previous reports demonstrated that these MRI changes represent a ring of demyelination, inflammation, and edema surrounding an area of gliosis, necrotic tissue, and macrophages (Narayana et al., 2004; Runge et al., 1997). We hypothesize that, at 21 days after SCI, the hypointense signal seen with MRI arises from hemosiderin containing macrophages and cellular debris that is surrounded by fluid, gliosis, and demyelinated white matter that together constitutes the hyperintense signal. The combined hypointense and hyperintense area may reflect the entire lesion cavity, recognizable by histopathology. In our images the border between the hyperintense rim and spared tissue was less well defined than the demarkation of the hypointense area, so we determined the volume of hypointense signal, rather than volume of the entire lesion cavity, throughout the lesion. We demonstrated a significant difference in volume of hypointense signal between the two study groups at 14 and 21 days after injury. It is probable that we underestimated the true lesion volume, since we did not include the area of hyperintense signal in this volume measurement; however, this did not affect the purpose of distinguishing two lesion severities.

The lesion volume difference most likely reflects the significant difference in lesion length that was produced by the two different contusion severities, shown by the various post mortem imaging modalities. The lesion length has been shown to be correlated to injury severity (Hackney et al., 1994). In addition, the hypointense area at the lesion epicenter at 21 days after injury in vivo, that was found to be significantly smaller in the $6.25 \mathrm{~g}$ $\mathrm{cm}$ group, may have contributed to the smaller volume seen in this group of animals. A significant difference in lesion area at lesion epicenter was not identified by post mortem MRI or histopathology, possibly due to substance within the lesion center being dissolved during tissue processing for histopathology. We can conclude that the area filled with blood products at epicenter was significantly smaller in the $6.25 \mathrm{~g}-\mathrm{cm}$ group, but once this is washed out during perfusion the remaining fluid filled cavity no longer is significantly different from that seen in the $25 \mathrm{~g}$ $\mathrm{cm}$ group. Histopathology determined the lesion area at epicenter in both groups to be significantly larger compared to MRI. This may be attributable to the fact we encompassed only the hypointense area in the MRI measurements and tissue changes that occurred during processing for histopathology. Furthermore, histopathology allows more detailed examination of sections and is likely to more precisely define the border between undamaged and damaged nervous tissue. Other studies in SCI suggest that lesion cavity may not be the best parameter for correlating injury impact parameters to behavior (Bresnahan et al., 1987, 1991); thus, more recent studies typically have used sparing of remaining and potentially functional tissue. In this study we did determine significantly more white matter sparing in the mildly injured animals by histopathology. In addition there were significantly more motor neurons remaining in the contralateral (uninjured) hemicord in the $6.25 \mathrm{~g}-\mathrm{cm}$ animals than in the $25 \mathrm{~g}-\mathrm{cm}$ group. Gensel et al. (2006) did not find a significant difference between their 6.25 and $12.5 \mathrm{~g}$ $\mathrm{cm}$ groups with respect to motor neuron counts. Accurate determination of tissue sparing and/or motor neuron count by MRI was not possible, but we showed high correlation of other injury severity parameters detectable by MRI, such as lesion length and cord volume, to behavior. 


\section{MRI IN LONGITUDINAL COMPARISON OF UNILATERAL CERVICAL SPINAL CORD INJURIES}

Here we demonstrate the capability of a combined 4.7T magnet and laboratory-built coil to acquire MR images that are of sufficient resolution to distinguish two different injury severities. The outcomes of this work suggest that in vivo longitudinal MRI can be used to not only assess evolution of injury site, but also to monitor experimental treatment strategies based on knowledge of MRI appearance of pathologic events. Moreover, MRI could be applied as a screening tool to either administer goaldirected therapies, or enable even group distribution, for example through quantification of swelling and edema. MRI has already proven to be useful for predicting neurological outcome and could be useful for categorizing patients for clinical studies. In this way some of the biological variability in SCI research may be reduced. The practicality of using sequential MRI with surface coils to study the evolution of SCI and treatments in rodents is likely to prove even more important as more advanced imaging and processing technologies are implemented. This should prove valuable in developing strategies for assessing the evolution and repair of SCI in the clinical setting.

\section{ACKNOWLEDGMENTS}

We would like to thank Rochelle Deibert, Crystal Forrider, Ryan Gilbert, and John Komon for their technical assistance. We would like to thank John Gensel for his help with behavioral analysis and Peter Wassenaar for his help with image acquisition. This work was supported by funds from the NIH (NS-31193), a grant from the New York State Center of Research Excellence (CoRE), and The Ohio State University, College of Medicine.

\section{REFERENCES}

Basso, D.M., Beattie, M.S., and Bresnahan, J.C. (1995). A sensitive and reliable locomotor rating scale for open field testing in rats. J. Neurotrauma 12, 1-21.

Berens, S.A., Colvin, D.C., Yu, C.G., Yezierski, R.P., and Mareci, T.H. (2005). Evaluation of the pathologic characteristics of excitotoxic spinal cord injury with MR imaging. Am. J. Neuroradiol 26, 1612-1622.

Bertelli, J.A., and Mira, J.C. (1993). Behavioral evaluating methods in the objective clinical assessment of motor function after experimental brachial plexus reconstruction in the rat. J. Neurosci. Methods 46, 203-208.

Bilgen, M., Abbe, R., Liu, S.J., and Narayana, P.A. (2000). Spatial and temporal evolution of hemorrhage in the hyperacute phase of experimental spinal cord injury: in vivo magnetic resonance imaging. Magn. Reson. Med. 43, 594-600.
Bilgen, M., Abbe, R., and Narayana, P.A. (2001a). Dynamic contrast-enhanced MRI of experimental spinal cord injury: in vivo serial studies. Magn. Reson. Med. 45, 614-622.

Bilgen, M., Dogan, B., and Narayana, P.A. (2002). In vivo assessment of blood-spinal cord barrier permeability: serial dynamic contrast enhanced MRI of spinal cord injury. Magn. Reson. Imaging 20, 337-341.

Bilgen, M., Elshafiey, I., and Narayana, P.A. (2001b). In vivo magnetic resonance microscopy of rat spinal cord at $7 \mathrm{~T}$ using implantable RF coils. Magn. Reson. Med. 46, 1250-1253.

Bilgen, M., and Narayana, P.A. (2001). A pharmacokinetic model for quantitative evaluation of spinal cord injury with dynamic contrast-enhanced magnetic resonance imaging. Magn. Reson. Med. 46, 1099-1106.

Boldin, C., Raith, J., Fankhauser, F., Haunschmid, C., Schwantzer, G., and Schweighofer, F. (2006). Predicting neurologic recovery in cervical spinal cord injury with postoperative MR imaging. Spine 31, 554-559.

Bresnahan, J.C., Beattie, M.S., Stokes, B.T., and Conway, K.M. (1991). Three-dimensional computer-assisted analysis of graded contusion lesions in the spinal cord of the rat. J. Neurotrauma $\mathbf{8 , 9 1 - 1 0 1 .}$

Bresnahan, J.C., Beattie, M.S., Todd, F.D., 3rd, and Noyes, D.H. (1987). A behavioral and anatomical analysis of spinal cord injury produced by a feedback-controlled impaction device. Exp. Neurol. 95, 548-570.

Chakeres, D.W., Flickinger, F., Bresnahan, J.C., Beattie, M.S., Weiss, K.L., Miller, C., and Stokes, B.T. (1987). MR imaging of acute spinal cord trauma. Am. J. Neuroradiol. 8, 5-10.

Deo, A.A., Grill, R.J., Hasan, K.M., and Narayana, P.A. (2006). In vivo serial diffusion tensor imaging of experimental spinal cord injury. J. Neurosci. Res. 83, 801-810.

Duncan, E.G., Lemaire, C., Armstrong, R.L., Tator, C.H., Potts, D.G., and Linden, R.D. (1992). High-resolution magnetic resonance imaging of experimental spinal cord injury in the rat. Neurosurgery 31, 510-519.

Elshafiey, I., Bilgen, M., He, R., and Narayana, P.A. (2002). In vivo diffusion tensor imaging of rat spinal cord at 7 T. Magn. Reson. Imaging 20, 243-247.

Falconer, J.C., Narayana, P.A., Bhattacharjee, M.B., and Liu, S.J. (1994). Quantitative MRI of spinal cord injury in a rat model. Magn. Reson. Med. 32, 484-491.

Gareau, P.J., Weaver, L.C., and Dekaban, G.A. (2001). In vivo magnetization transfer measurements of experimental spinal cord injury in the rat. Magn. Reson. Med. 45, 159-163.

Gensel, J.C., Tovar, C.A., Hamers, F.P., Deibert, R.J., Beattie, M.S., and Bresnahan, J.C. (2006). Behavioral and histological characterization of unilateral cervical spinal cord contusion injury in rats. J. Neurotrauma 23, 36-54.

Gruner, J.A. (1992). A monitored contusion model of spinal cord injury in the rat. J. Neurotrauma 9, 123-128. 


\section{MIHAI ET AL.}

Hackney, D.B., Ford, J.C., Markowitz, R.S., Hand, C.M., Joseph, P.M., and Black, P. (1994). Experimental spinal cord injury: MR correlation to intensity of injury. J. Comput. Assist. Tomogr. 18, 357-362.

Miyanji, F., Furlan, J.C., Aarabi, B., Arnold, P.M., and Fehlings, M.G. (2007). Acute cervical traumatic spinal cord injury: MR imaging findings correlated with neurologic outcome-prospective study with 100 consecutive patients. Radiology 243, 820-827.

Narayana, P., Abbe, R., Liu, S.J., and Johnston, D. (1999). Does loss of gray- and white-matter contrast in injured spinal cord signify secondary injury? In vivo longitudinal MRI studies. Magn. Reson. Med. 41, 315-320.

Narayana, P.A., Grill, R.J., Chacko, T., and Vang, R. (2004). Endogenous recovery of injured spinal cord: longitudinal in vivo magnetic resonance imaging. J. Neurosci. Res. 78, 749-759.

National Spinal Cord Injury Statistical Center. (2006). Facts and Figures at a Glance. University of Alabama, Birmingham.

Ohta, K., Fujimura, Y., Nakamura, M., Watanabe, M., and Yato, Y. (1999). Experimental study on MRI evaluation of the course of cervical spinal cord injury. Spinal Cord 37, 580-584.

Pearse, D.D., Lo, T.P., Jr., Cho, K.S., Lynch, M.P., Garg, M.S., Marcillo, A.E., Sanchez, A.R., Cruz, Y., and Dietrich, W.D. (2005). Histopathological and behavioral characterization of a novel cervical spinal cord displacement contusion injury in the rat. J. Neurotrauma 22, 680-702.

Popovich, P.G., Guan, Z., Wei, P., Huitinga, I., Van Rooijen, N., and Stokes, B.T. (1999). Depletion of hematogenous macrophages promotes partial hindlimb recovery and neuroanatomical repair after experimental spinal cord injury. Exp. Neurol. 158, 351-365.

Popovich, P.G., Horner, P.J., Mullin, B.B., and Stokes, B.T. (1996). A quantitative spatial analysis of the blood-spinal cord barrier. I. Permeability changes after experimental spinal contusion injury. Exp. Neurol. 142, 258-275.

Runge, V.M., Wells, J.W., Baldwin, S.A., Scheff, S.W., and Blades, D.A. (1997). Evaluation of the temporal evolution of acute spinal cord injury. Invest. Radiol. 32, 105-110.

Schallert, T., Hernandez, T.D., and Barth, T.M. (1986). Recovery of function after brain damage: severe and chronic disruption by diazepam. Brain Res. 379, 104-111.
Schwartz, E.D. (2005). MRI and the evaluation of the blood-spinal cord barrier following injury. Am. J. Neuroradiol. 26, 1609-1610.

Schwartz, E.D., and Hackney, D.B. (2003). Diffusion-weighted MRI and the evaluation of spinal cord axonal integrity following injury and treatment. Exp. Neurol. 184, 570-589.

Selden, N.R., Quint, D.J., Patel, N., D’Arcy, H.S., and Papadopoulos, S.M. (1999). Emergency magnetic resonance imaging of cervical spinal cord injuries: clinical correlation and prognosis. Neurosurgery 44, 785-792.

Silver, X., Ni, W.X., Mercer, E.V., Beck, B.L., Bossart, E.L., Inglis, B., and Mareci, T.H. (2001). In vivo ${ }^{1} \mathrm{H}$ magnetic resonance imaging and spectroscopy of the rat spinal cord using an inductively-coupled chronically implanted RF coil. Magn. Reson. Med. 46, 1216-1222.

Soblosky, J.S., Song, J.H., and Dinh, D.H. (2001). Graded unilateral cervical spinal cord injury in the rat: evaluation of forelimb recovery and histological effects. Behav. Brain Res. 119, 1-13.

Waibl, H. (1973). Zur Topographie der Medulla Spinalis der Albinoratte (Rattus norvegicus). Springer Verlag: Berlin.

Weber, T., Vroemen, M., Behr, V., Neuberger, T., Jakob, P., Haase, A., Schuierer, G., Bogdahn, U., Faber, C., and Weidner, N. (2006). In vivo high-resolution MR imaging of neuropathologic changes in the injured rat spinal cord. Am. J. Neuroradiol. 27, 598-604.

Weirich, S.D., Cotler, H.B., Narayana, P.A., Hazle, J.D., Jackson, E.F., Coupe, K.J., McDonald, C.L., Langford, L.A., and Harris, J.H., Jr. (1990). Histopathologic correlation of magnetic resonance imaging signal patterns in a spinal cord injury model. Spine 15, 630-638.

Whetstone, W.D., Hsu, J.Y., Eisenberg, M., Werb, Z., and Noble-Haeusslein, L.J. (2003). Blood-spinal cord barrier after spinal cord injury: relation to revascularization and wound healing. J. Neurosci. Res. 74, 227-239.

Address reprint requests to: Michael Beattie, Ph.D.

Brain and Spinal Injury Center Department of Neurological Surgery University of California, San Francisco 1001 Potrero Avenue San Francisco, CA 94110

E-mail: michael.beattie@ucsf.edu 